\title{
Trypanosoma evansi: A Qualitative and Quantitative Ultrastructural Analysis of the Spleen during Experimental Murine Infections
}

\author{
Lucia Proietti d'Empaire1, Félix Tejero², Hector J. Finol'3, Pedro M. Aso ${ }^{4}$, \\ Antonio Roschman-Gonzalez ${ }^{3 *}$ \\ ${ }^{1}$ Centro de Biología Estructural, Instituto Venezolano de Investigaciones Científicas, Caracas, Venezuela \\ ${ }^{2}$ Instituto de Zoología y Ecología Tropical, Facultad de Ciencias, Universidad Central de Venezuela, Caracas, Venezuela \\ ${ }^{3}$ Centro de Microscopía Electrónica, Facultad de Ciencias, Universidad Central de Venezuela, Caracas, Venezuela \\ ${ }^{4}$ Departamento de Biología Celular, Universidad Simón Bolívar, Caracas, Venezuela \\ Email: ${ }^{\star}$ roschman@gmail.com
}

How to cite this paper: d'Empaire, L.P., Tejero, F., Finol, H.J., Aso, P.M. and Roschman-Gonzalez, A. (2020) Trypanosoma evansi: A Qualitative and Quantitative Ultrastructural Analysis of the Spleen during Experimental Murine Infections. Microscopy Research, 8, 31-42.

https://doi.org/10.4236/mr.2020.83003

Received: July 6, 2020

Accepted: July 27, 2020

Published: July 30, 2020

Copyright $\odot 2020$ by author(s) and Scientific Research Publishing Inc. This work is licensed under the Creative Commons Attribution International License (CC BY 4.0).

http://creativecommons.org/licenses/by/4.0/

\begin{abstract}
A murine model is used to study qualitatively and quantitatively the splenic ultrastructural changes induced by two Trypanosoma evansi strains derived from naturally infected local equine hosts (Equusasinus and E. caballus); $T$. evansi causes ultrastructural modifications in the spleen of the infected mice. The modifications include tissular disorganization, fibrosis, mitochondrial swelling, apoptosis and necrosis. The initial phases of the infection are quite similar, whereas the final phases differ qualitatively depending on the strain's source. The ultrastructural quantitative changes were studied in the reticular splenocytes covering alterations in the area of the cytoplasm and nucleus. Analysis of the results shows the induction of various splenic alterations caused by local T. evansi strains. Also, it was documented that discriminative time modulation, as well as progressive tissular, cellular and subcellular changes, are more associated with derived infections from $E$. caballus strain.
\end{abstract}

\section{Keywords}

Trypanosoma evansi, Spleen, Ultrastructure, Mouse

\section{Introduction}

Trypanosoma evansi is the etiologic agent of equine trypanosomosis in the savannas of Venezuela. The equines, in particular horses, are widely used in the extensive bovine farming and equine health is therefore an important factor in bovine production. 
While there is some understanding about the ultrastructural pathology of the infections induced by local T. evansi isolates [1] [2] [3] [4], it should be noted that an important genetic [5] and parasitological [6] variability has been demonstrated in the behavior of different strains [6], nothing is known about its effects in the spleen, and much less still regarding the comparative action of two strains of equine origin upon this organ.

The spleen is a lymphatic organ interposed in the blood stream having three major functions: phagocytosis of erythrocytes and cell debris from the blood stream (producing foci of hemopoiesis when erythrocytes are needed); supply of lymphocytes, plasma cells and hence antibodies for the cellular and humoral specific immune defenses; ability to expel contained blood to meet increased circulatory demands [7]. So, studying the effects of an archetypal blood trypanosome upon this organ represents a significant contribution to the understanding of the biology of T. evansi.

In order to demonstrate the spleen sequential ultrastructural modifications under $T$. evansi stress and comparatively evaluate the induced subcellular changes, we established experimental murine infections with two equine strains of local T. evansi, one obtained from Equusasinus and the other derived from $E$. caballus.

\section{Materials and Methods}

\subsection{Obtaining T. evansi Trypomastigotes from E. asinus, E. cabalus}

T. evansi trypomastigotes derived from a naturally infected E. asinus at "Terecay" cattle ranch (Venezuela, Guarico State, Calabozo City) and a naturally infected E. caballus at "Mantecal" town Venezuela, Apure State, Mantecal City) were stabilized in immunosuppressed Balb/C mice [8], separated from erythrocytes [9] and maintained in liquid $\mathrm{N}_{2}$ [10] until use.

\subsection{Parasites Inoculation and Control Group}

The experimental design consisted of three groups of 10 mice each (NMRI female $20 \mathrm{~g}$ of body weight): Group 1, mice intradermally infected with T. evansi obtained from E. asinus. Group 2, mice intradermally infected with T. evansi from E. caballus. And Group 3, control mice (intradermally inoculated with saline solution). Groups 1 and 2 mice were infected with 20 trypomastigotes suspended in $0.1 \mathrm{ml}$ of saline phosphate solution $0.02 \mathrm{M}+1 \%$ glucose, $\mathrm{pH} 7.0$ (GSPB).

\subsection{Parasithemia, Hematocrit and Surgical Spleen Ablation}

From the third day post infection, interday and ramdoly, we chose one mouse of each group (mice infected with $E$. asinus strain, mice infected with $E$. cabalus strain, and mice inoculated with saline. Control group).

To count the parasites, we used a hemocytometercounting chamber with Neubauer rulings and serial GSPB dilutions to obtain the desired concentration. 
Additionally, to these mice the hematocrit was recorded. To collect spleen tissue samples, mice died under the action of the anesthesia. By means of ether atmosphere surgical ablation, the spleen was removed and systematically sectioned in $2 \mathrm{~mm} 3$ portions.

\subsection{Electron Microscopy Techniques}

The spleen fragments were fixed with Karnovsky solution (2.5\% glutaraldehyde, 37\% formaldehyde in Millonig buffer; $\mathrm{pH}$ 7.4, $320 \mathrm{mOsm}$ ), washed in Millonig buffer, post-fixed in $1 \%$ osmium tetroxide solution (same buffer, $\mathrm{pH}$ and osmolarity), submerged in distilled water and dehydrated in an ethanol series of increasing concentrations $(50 \%-100 \%)$. Soon after, the pieces were infiltrated with propylene oxide and included in epoxy resin [11]. The contrasted [12] [13] ultrathin sections $(60-80 \mathrm{~nm})$ were observed with an electron microscope (CM-10; Philips, The Netherlands).

\subsection{Ultrastructural Quantitative Analysis and Statistical Analysis}

The obtained micrographs were digitalized, qualitatively analyzed and quantitatively studied. Focusing on the reticular splenocytes (RS), the cytoplasmic area (CA) and the nuclear area (NA) of 50 cells were calculated by means of ImageJ software. In order to look at the problem from a quantitative multivariate scope, the length of both major and minor nucleus axes, the mitochondrial area, and the nucleus area/cytoplasm area ratio were also calculated (data not shown).

After corroborating the assumptions of normality and homogeneity of variance, the obtained numeric data were quantitatively processed by means of 2-way analysis of the variance (ANOVA) [14], simple linear regression (SLR) [14] using STATISTICA V.8.0 software and correspondence analysis (COA) [15], employing MVSP V.2.1 software. The graphic is presented as a standard error plot and COA as continuous clouds of points. All tests were done at $\mathrm{p}<$ 0.05. Duplicate experiments were repeated three times with different batches of mice and parasites. The investigation complied with the norms set out in the Guide for Care and Use of Laboratory Animals, published by the US National Institute of Health (NIH publication No. 85-23, revised 1985).

\section{Results and Discussion}

Since the parasitaemia $(\mathrm{P})$ and the hematocrit $(\mathrm{H})$ are relevant indicators evaluating the general health condition of a mammal our first approach was through these variables.

The quantitative analysis indicated significative differences in $P$ depending upon the T. evansi origin (i.e. E. asinus or E. caballus) $(p=0.00)$, the time $(p=0.00)$ and the strainorigin-time interaction $(p=0.00)$. In other respects, the test of the $\mathrm{F}$ values demonstrates significative differences linked to the strainorigin $(p=$ $0.00)$, time $(p=0.00)$ and the strain origin-time interaction $(p=0.00)$ (Table 1$)$. 
Table 1. Analysis of the variance for the parasitaemia, hematocrit, and cytoplasmic and nuclear area of reticular splenocytes.

\begin{tabular}{|c|c|c|c|c|c|}
\hline \multirow{2}{*}{$\begin{array}{l}\text { Source of } \\
\text { variation }\end{array}$} & \multirow{2}{*}{$\begin{array}{l}\text { Degrees of } \\
\text { freedom }\end{array}$} & \multicolumn{4}{|c|}{ F-value } \\
\hline & & Parasitaemia & Hematocrit & $\begin{array}{c}\text { Cytoplasmic } \\
\text { area }\end{array}$ & Nuclear area \\
\hline Strains & 2 & $495.44^{*}$ & $12.93^{*}$ & $495.44^{*}$ & $495.44^{\star}$ \\
\hline Time (days) & 7 & $69.09^{*}$ & $15.15^{*}$ & $69.09^{*}$ & $69.09^{*}$ \\
\hline Interaction & 14 & $19.77^{\star}$ & 0.25 & $19.77^{\star}$ & $19.77^{\star}$ \\
\hline
\end{tabular}

*Significance $p<0.05$.

Otherwise, the earliest indicator of spleen deterioration in mice infected with the horse derived strain was defined by electron dense cytoplasm from day 3 post-infection (Figure 1).

In general, the qualitatively splenic ultrastructural change was progressive, being characterized by a high level of tissular disorganization and fibrosis depicted by apoptotic cells, necrotic cells and cellular destruction (including RS). The subcellular characteristics of the change comprise pyknotic and irregular nuclei, hyperchromatism, altered and/or peripheral chromatin, electron dense cytoplasm, swelling mitochondria, distended cisternae of the Golgi apparatus, autophagosomes, and apoptotic bodies.

Trypanosomes were seen in the spleen from day 9 and day 7 in mice infected with the strains obtained from $E$. asinus and $E$. caballus, respectively, whereas subcellular anomalies were patent from day 7 in mice infected with the donkey strain and from day 3 in animals inoculated with the horse derived parasites.

The micrographic record makes clear the changes and the trypomastigote's presence. Indeed, from day 7 post-infection the spleen of the animals inoculated with the donkey strain showed the first signs of damage, being characterized by macrophages exhibiting electron transparent cytoplasm and swelling mitochondria (Figure 2).

As the experimental infection progresses the parasites show up in the spleen. The trypanosomes were evident from day 7 post-infection in the spleen of the animals inoculated with the horse derived strain, and from day 9 post-infection with the strain obtained from $E$. asinus (Figure 3).

No particular signs were evident in the ultrastructural morphology of the parasites, defined by the usual identifiable mammal's trypanosome characteristics.

The infection's final phases are distinguished by patent tissue disorganization and deep subcellular changes. Infections initiated with parasites obtained from $E$. asinus are characterized by apoptotic cells contained in disorganized tissue whereas those begun with the trypanosomes derived from $E$. caballus are portrayed by generalized vacuolization as well as a high degree of cellular destruction. 
The quantitative analysis of the RS ultrastructure demonstrated changes. The cytoplasm area showed definite significant modifications. The ANOVA establishes that the differences seen in the cytoplasmic area are due to the isolate source $(p=0.00)$, the time $(p=0.00)$ and the interaction isolate's source-time ( $p$ $=0.00$ ) (Table 1). Additionally, the SLR demonstrates the velocity of change being more rapid in mice infected with the $T$. evansi derived from $E$. caballus than those inoculated with the E. asinus (Figure 4).

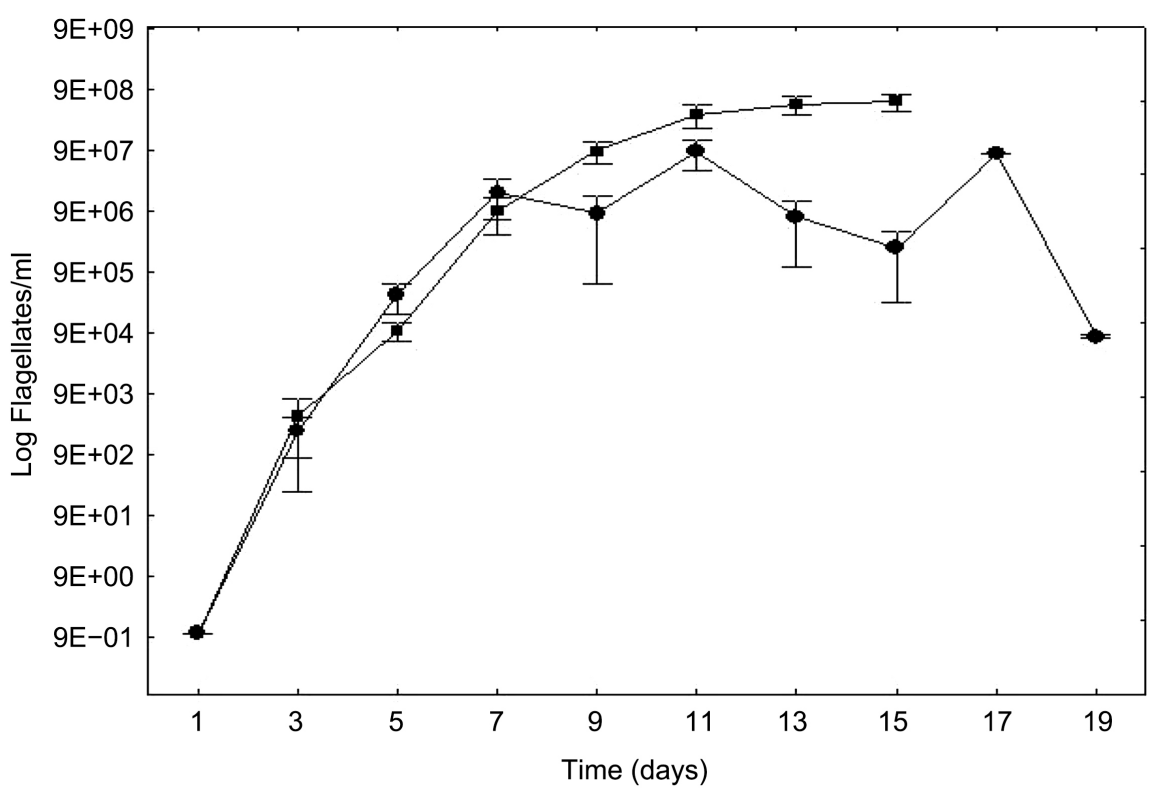

(a)

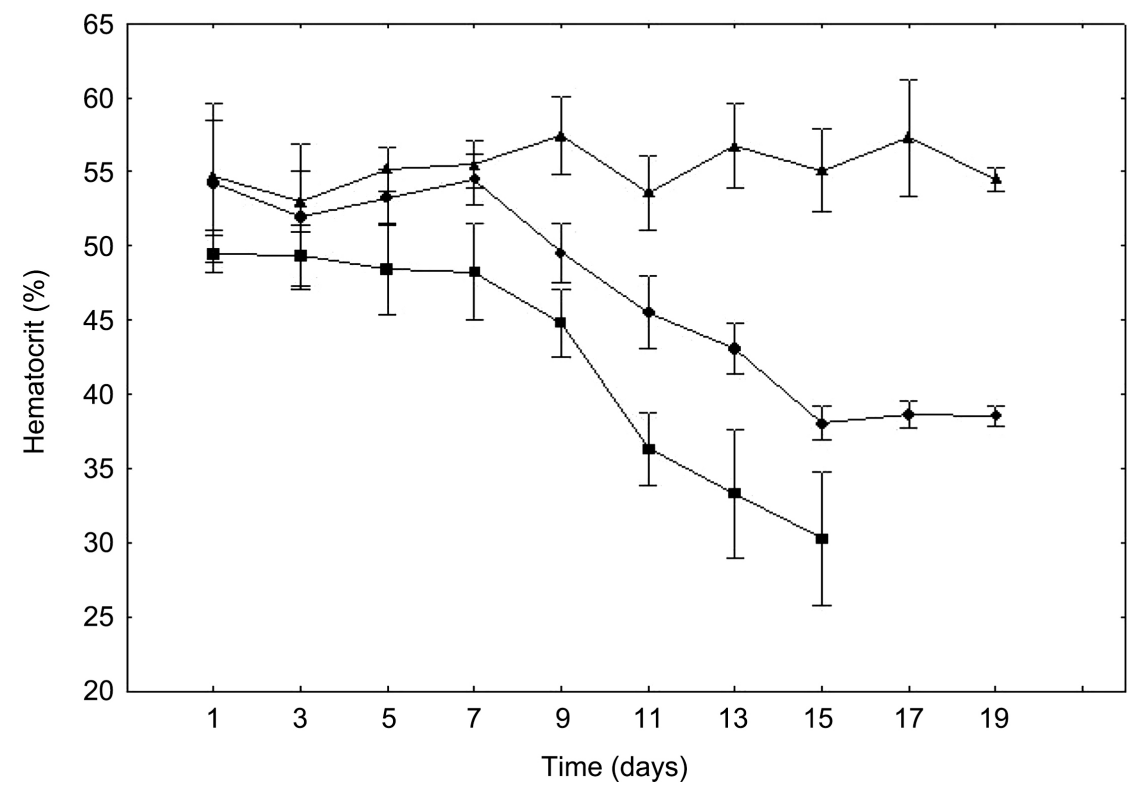

(b)

Figure 1. (a) Course of the parasitaemia of NMRI mice infected with Trypanosoma evansi: • T. evansi derived from E. asinus, a: T. evansi from E. caballus, control groups did not develop parasitaemia; (b) Course of the hematocrit of NMRI mice infected with $T$. evansi. •: T. evansi derived from E. asinus, n: T. evansi from E. caballus, $\mathbf{\Delta}$ : control. 


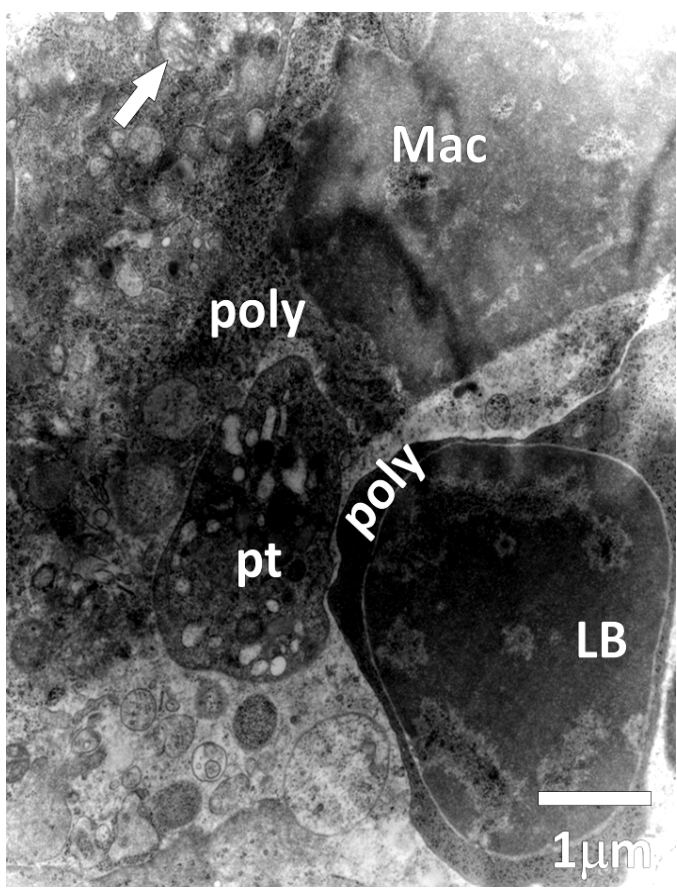

Figure 2. The micrograph shows a section of the spleen 7 days' post-infection. A platelet (pt) is surrounded by a macrophage (Mac) exhibiting a swollen mitochondrion (arrow), hyperchromatic nucleus and abundant polysomes (poly). A lymphoblast (LB) is also seen displaying the cytoplasm typically upholstered by polysomes (poly); its electron density suggests an initial apoptotic phase. Parasites derived from $E$. asinus.

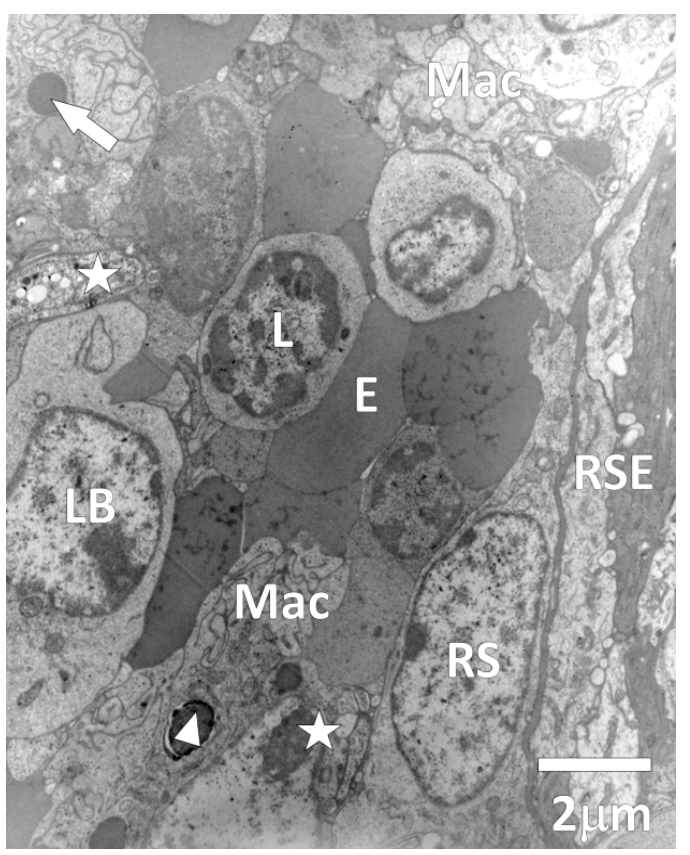

Figure 3. The micrograph shows an ultrathin section of the spleen 9 days post-infection. The micrograph shows two trypanosomes (star), erythrocytes (E), lymphocytes (L) plus reticular splenocytes (RS) and extensions of a reticular splenocyte (RSE). Lymphoblasts (LB) and macrophages (Mac) are seen. In addition, the micrograph displays autophagic vacuoles (arrow) as well as a body surrounded by a membrane containing, in appearance, phagocytic debris (triangle). Parasites derived from E. asinus. 


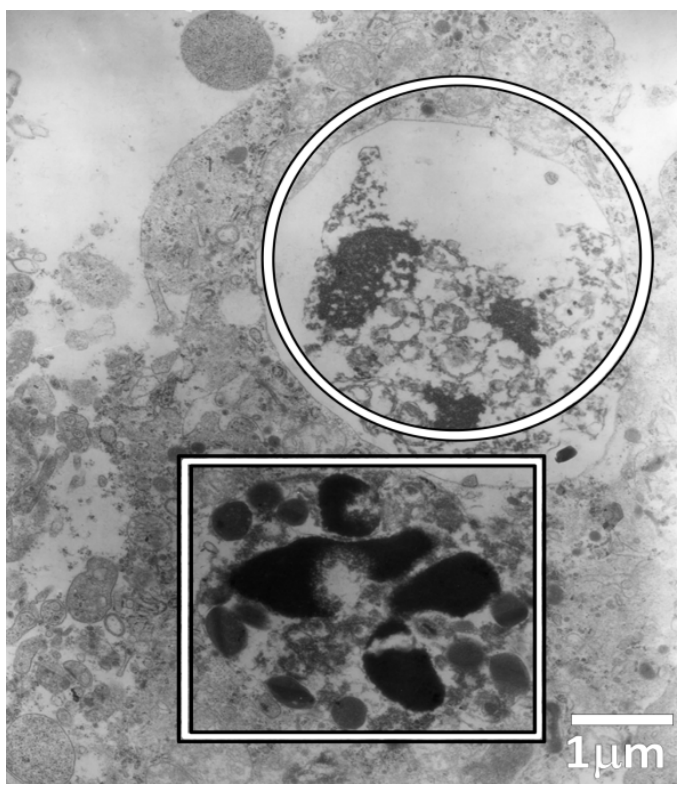

Figure 4. The micrograph shows an ultrathin section of the spleen 15 days post-infection. A macrophage containing bodies of different form, size and electrondensity is evident (circle), together with phagocytic debris (rectangle). The generalized vacuolization is evidence bytissular disorganization. Parasites derived from $E$. caballus.

Such an assertion is corroborated by the high predictability of the proposed linear model since the regression coefficients (r) are -0.9200 and -0.9653 for $E$. asinus and $E$. caballus, respectively. These regression coefficients are in turn different to that of the control which was a consistent -0.9071 (Table 2).

In other aspects, the behavior of the variable nucleus area exhibits important changes depending on the parasite's source, the time and the isolate source-time interaction. The ANOVA establishes that the probability level associated with the observed differences are $p=0.00$ for the isolate source, $p=0.00$ for the time and $p=0.00$ for the interaction of the isolate source and the time; the high $\mathrm{F}$ values, particularly for the isolate origin, further emphasize these differences (Table 1). The analysis also demonstrated the significance of the change for each parasite isolate and the linearity of the nuclear area changes, which were faster in the animals infected with $T$. evansi isolate derived from $E$. caballus than that derived from $E$. asinus (Table 2). The preceding statement is confirmed by the high predictability level of the linear model since the regression coefficients ( $r$ ) are -0.9968 and -0.9943 for $E$. asinus and E. caballus derived isolates, respectively; the control's behavior is totally different (Table 2).

The comparative analysis of the cytoplasmic and nuclear areas of the RS demonstrates a significant decrease of the cell area in comparison with the controls. The statistical characterization shows a more accentuated splenic alteration in the mice infected with parasites derived from E. caballus.

The Correspondence Analysis (COA) is a descriptive/exploratory multivariate technique used to graphically analyze the dependency/independency relations in a set of categorical variables. The model bears clouds of points corresponding to 
Table 2. Simple lineal regression for the cytoplasmic area $(\mathrm{Ca})$ and nuclear area $(\mathrm{Na})$ of the reticular splenocytes.

\begin{tabular}{cccc}
\hline Strains & Equation & $\mathrm{r}$ & $p$ \\
\hline Equus asinus & $\mathrm{Ca}=26.37-1.21 \times$ time & -0.9200 & $0.0094^{\star}$ \\
& $\mathrm{Na}=30.49-1.93 \times$ time & -0.9968 & $0.0049^{\star}$ \\
Equus caballus & $\mathrm{Ca}=22.88-1.83 \times$ time & -0.9653 & $0.0347^{\star}$ \\
& $\mathrm{Na}=17.71-1.20 \times$ time & -0.9943 & $0.0470^{\star}$ \\
Control & $\mathrm{Ca}=18.98-0.02 \times$ time & -0.9071 & $0.0276^{\star}$ \\
& $\mathrm{Na}=4.60-0.02 \times$ time & 0.8955 & $0.0483^{\star}$ \\
\hline
\end{tabular}

the numerical magnitudes of the variables in the original hyperspace were downsized and plotted in a new reduced 2-dimensional space. Superimposing clouds signify identity while separated clouds signify disparity; the closer or separated the clouds are, the more similar or dissimilar are the processes that they represent. Furthermore, the uninterrupted connection of a particular cloud is interpreted as the continuity of the represented phenomenon. The procedure is able to compress the original eight variables hyperspace (including the nucleus area/cytoplasm area ratio) to a Cartesian coordinates graph comprising two axes. The results conclusively show significant change in time and clearly define discrimination between the experimental and the control groups. The tendencies of change sequence in the experimental groups are relatively similar in the infection's initial phases since the clouds of points exhibit some kind of parallelism behavior. On the other hand, the late phases are clearly dissimilar; on the basis of the cloud form and its distribution on the plane it can be said that both tendencies are dissimilar and very different to the control group. The COA model undoubtedly distinguishes the change peculiarities between the mice infected with $T$. evansi derived from E. asinus or E. caballus as well as with the controls (Figure 5).

The host-parasite relationship is a complex interactive system becoming evident through multiple characteristics including biochemical, physiological, parasitological, and ecological adaptations complemented with a variety of adaptive mechanisms, which empower the parasite to evade the host immune response.

T. evansi infection shows different anatomopathological episodes, including splenomegaly [16] [17] [18] [19] [20]. In this aspect, our results evidenced ultrastructural splenic alterations that caused different parasitological expressions, depending on the T. evansi source (E. asinus or E. caballus). The parasitemic dissimilarity idea provided by the curve's outlines was confirmed by the ANOVA. Furthermore, the ANOVA also quantitatively confirmed the differences showed in the hematocrit curves. Depending on the infective source the health indicators (parasitaemia and hematocrit) exhibited different behaviors. This may suggest a relationship between the source of the trypanosome (in terms of adaptation) and its ability to colonize and exploit new hosts successfully. 


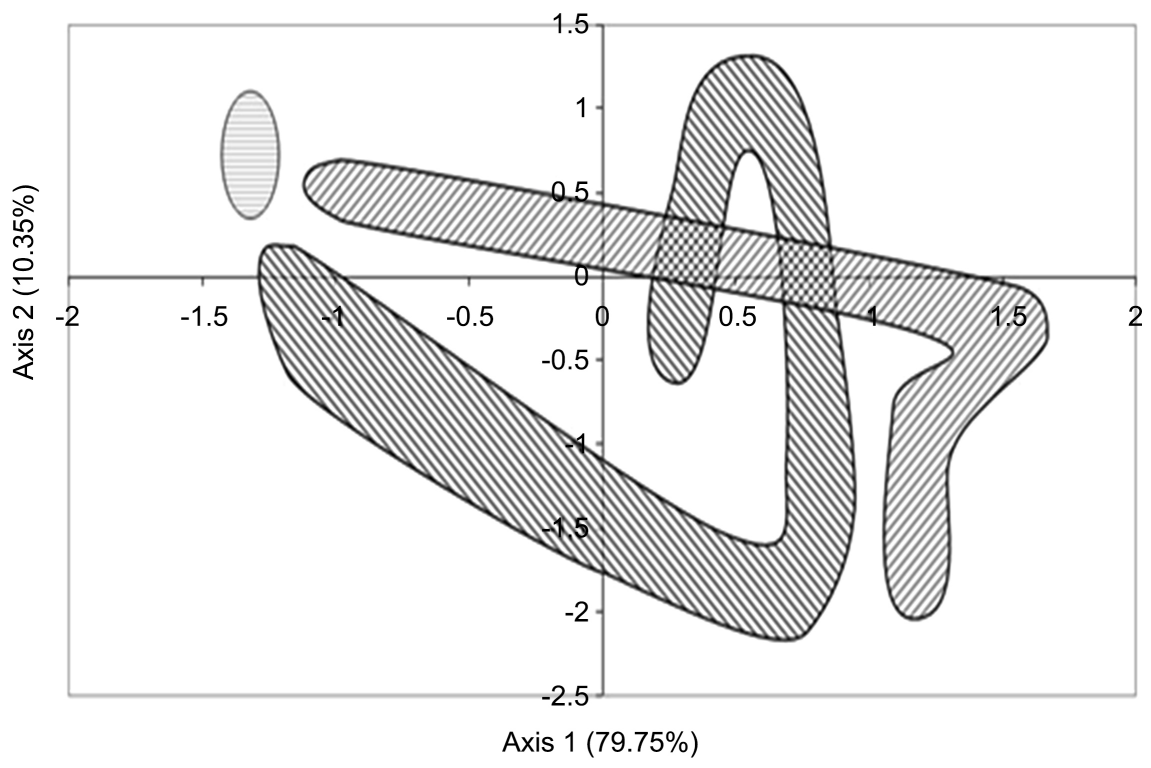

Figure 5. The graph shows the two-dimensional representation of the Correspondence Analysis. The Correspondence Analysis has been represented in a Cartesian plane where Axis 1 contains $79.75 \%$ of the system's total variance and Axis $2 \%, 10 \%, 35 \%$. The original eight variables hyperdimensional space reduction is restrained to three clouds of points corresponding to the T. evansi strains and the controls. E. asinus derived strain group (clockwise striped area); T. evansi from E. caballus group (counter clockwise striped area); and control group (horizontally striped area).

The splenic changes, besides the parasite's action by means of a possible capability for particular proteolytic secretions [21], could be related to T. evansi induced hepatic alteration [3], since liver deterioration can raise the portal pressure. Indeed, advanced hepatomegaly increases the portal flux and makes it possible for blood to flow through collateral systems via portal and cava veins [22]. The portal flux increments are determined by vasodilatation of the splanchnic tissue (stomach, intestine, pancreas and spleen) which in turn permits an increase in the blood flow to these organs [23].

The micrographic results also showed intrasplenic trypanosomes. In the absence of conclusive evidence, and since $T$. evansi is a typical monomorphic blood trypanosome, we suggest that the presence of the parasite in the spleen is a result of circulating trypomastigotes, driven into the splenic parenchyma by the blood circulation itself.

The first qualitative transmission electron microscopy indication of damage is the presence of a considerable amount of splenic debris. The presence of such remains could be due to the erythrophagocytosis and cell debris phagocytosis occurring in the infected spleen. The occurrence of parasite induced pathological processes can be demonstrated by comparing the observed ultrastructural changes and RS quantitative changes with those seen in the controls. However, individual ultrastructural modifications are not differentiable by particular characteristics. The observed differences in the elapsed time for the changes to appear and the velocity of the processes itself, expressed as the slope of the SLR 
straight lines, could suggest intrinsic parasite dissimilarities since other authors [24] have established that morphologic contrasts among $T$. evansi populations are able to express particular biochemical, physiological and/or pathologic behaviors. The differential expression of the equations of change in the cytoplasmic and nuclear RS areas provided by the SLR model indicates the disparity of the described phenomena.

The quantitative analysis of the RS also reveals subtle differences in the change process of the investigated variables. The ANOVA and SLR analyses demonstrated dissimilarities in the source of variation and the experimental infection's elapsed time by parasite origin (donkey or horse). Previously we have proved [25] the quantitative relationship between the dimensionality of the T. evansi trypomastigotes and some hematological attributes of mice experimentally infected with these strains

\section{Conclusions}

By way of conclusion we can say that the qualitative and quantitative expressions of the pathological processes induced by local $T$. evansi derived from $E$. asinus and $E$. caballus differ in the velocity of change of RS through particular tendency patterns, and particularly in ultrastructural characteristics during the phases of late infection. The results suggest differential pathogenicity depending on the trypanosome's source. The COA demonstrates a succession of changes and gives evidence of different pathological behaviors depending on the trypanosome's source (E. asinus or E. caballus). And finally, the analysis of the results demonstrates induction of various splenic ultrastructural alterations provoked by different local T. evansi strains with more stress consistently associated to E. caballus parasites.

\section{Acknowledgements}

In memoriam of Professor Hector J. Finol (1945-2020), master and friend.

The financial support of FONACIT is gratefully acknowledged (Project G98-03462).

\section{Conflicts of Interest}

The authors declare no conflicts of interest regarding the publication of this paper.

\section{References}

[1] Quiñones Mateu, M.E., Finol, H.J., Sucre, L.E. and Torres, S.H. (1994) Muscular Changes in Venezuelan Wild Horses Naturally Infected with Trypanosoma evansi. Journal of Comparative Pathology, 110, 79-89. https://doi.org/10.1016/S0021-9975(08)80272-1

[2] Finol, H.J., Boada-Sucre, A., Rossi, M. and Tejero, F. (2001) Skeletal Muscle Ultrastructural Pathology in Mice Infected with Trypanosoma evansi. Journal of Submicroscopic Cytology and Pathology, 33, 65-71. 
[3] Herrera, H.M., Alessi, A.C., Marques, L.C., Santana, A.E., Aquino, L.P.C.T., Menezes R.F., Moraes, M.A.V. and Machado, R.Z. (2002) Experimental Trypanosoma evansi Infection in South American Coati (Nasua nasua): Hematological, Biochemical and Histopathological Changes. Acta Tropica, 81, 203-210. https://doi.org/10.1016/S0001-706X(01)00204-2

[4] Tejero, F., Brun, S., Roschman-González, A., Perrone-Carmona, T.M., Aso, P.M., Velasco, E. and Finol, H.J. (2009) Trypanosome evansi: Analysis of the Ultrastructural Change in Hepatic Cells during Murine Experimental Infections. Acta Microscopica, 18, 28-32.

[5] Perrone, T.M., Gonzatti, M.I., Villamizar, G., Escalante, A. and Aso, P.M. (2009) Molecular Profiles of Venezuelan Isolates of Trypanosoma sp. by Random Amplified Polymorphic DNA Method. Veterinary Parasitology,161,194-200 https://doi.org/10.1016/j.vetpar.2009.01.034

[6] Parra-Gimenez, N. and Reyna-Bello, A.(2019) Parasitological, Hematological, and Immunological Response of Experimentally Infected Sheep with Venezuelan Isolates of Trypanosoma evansi, Trypanosoma equiperdum, and Trypanosoma vivax. Journal of Parasitology Research, 2019, Article ID: 8528430. https://doi.org/10.1155/2019/8528430

[7] Steiniger, B. and Barth, P. (2000) Microanatomy and Function of the Spleen. Springer-Verlag, Berlin, 45-49. https://doi.org/10.1007/978-3-642-57088-9

[8] Rossi, M., Boada-Sucre, A., Finol, H.J., Tejero, F., Bello, B., Aso, P.M. and Hernandez, G. (1999) Ultrastructural Alterations in the Adrenal Gland Cortex of Mice Experimentally Infected with a Venezuelan Isolate of Trypanosoma evansi. Journal of Submicroscopic Cytology and Pathology, 31, 509-513.

[9] Woo, P.T.K. (1970) The Haematocrit Centrifuge Technique for the Diagnosis of African Trypanosomiasis. Acta Tropica, 27, 384-386.

[10] Schuster, J.P., Mehlhorn, H. and Raether, W. (1996) Ultrastructural Changes on Various Trypanosoma spp. after a 30-Year Storage Period in Liquid Nitrogen. Parasitology Research, 82, 720-726. https://doi.org/10.1007/s004360050191

[11] Luft, J.H. (1961) Improvements in Epoxy Resin Embedding Methods. The Journal of Biophysical and Biochemical Cytology, 9, 409-414. https://doi.org/10.1083/jcb.9.2.409

[12] Reynolds, E.S. (1963) The Use of Lead Citrate at High pH as an Electron-Opaque Stain in Electron Microscopy. Journal of Cell Biology, 17, 208-212. https://doi.org/10.1083/jcb.17.1.208

[13] Watson, M.L. (1958) Staining of Tissue Sections for Electron Microscopy with Heavy Metals. The Journal of Biophysical and Biochemical Cytology, 4, 475-478. https://doi.org/10.1083/jcb.4.4.475

[14] Chou, Y.L. (1975) Statistical Analysis. Holt, Rinehart \& Winston, New York, 343-355.

[15] Chatfield, C. and Collins, A.J. (1980) Introduction to Multivariate Analysis. Chapman\& Hall, London, 189-229.

[16] Audu, P.A., Esievo, K.A., Mohammed, G. and Ajanusi, O.J. (1999) Studies of Infectivity and Pathogenicity of an Isolate of Trypanosoma evansi in Yankasa Sheep. Veterinary Parasitology, 86, 185-190. https://doi.org/10.1016/S0304-4017(99)00141-7

[17] Biswas, D., Choudhury, A. and Misra, K.K. (2001) Histopathology of Trypanosoma (Trypanozoon) evansi Infection in Bandicoot Rat. I. Visceral Organs. Experimental Parasitology, 99, 148-159. https://doi.org/10.1006/expr.2001.4664 
[18] Damayanti, R., Graydon, R.J. and Ladds, P.W. (1994) The Pathology of Experimental Trypanosoma evansi Infection in the Indonesian Buffalo (Bubalus bubalis). Journal of Comparative Pathology, 110, 237-252. https://doi.org/10.1016/S0021-9975(08)80277-0

[19] Gill, B.S. (1977) Trypanosomes and Trypanosomiases of Indian Livestock. Indian Council of Agricultural Research, New Delhi, 1-136.

[20] Uche, U.E. and Jones, T.W. (1994) Protection Conferred by Trypanosoma evansi Infection against Homologous and Heterologous Trypanosome Challenge in Rabbits. Veterinary Parasitology, 52, 21-35. https://doi.org/10.1016/0304-4017(94)90032-9

[21] Galindo, B. and Freeman, J.A. (1963) Fine Structure of Splenic Pulp. The Anatomical Record, 147, 25-41. https://doi.org/10.1002/ar.1091470105

[22] Galindo, B. and Imaeda, T. (1962) Electron Microscope Study of the White Pulp of the Mouse Spleen. The Anatomical Record, 143, 399-415. https://doi.org/10.1002/ar.1091430410

[23] Magalotti, D., Marchesini, G., Ramilli, S., Berzigotti, A., Bianchi, G. and Zoli, M. (2004) Splanchnic Haemodynamics in Non-Alcoholic Fatty Liver Disease: Effect of a Dietary/Pharmacological Treatment. A Pilot Study. Digestive and Liver Disease, 36, 406-411. https://doi.org/10.1016/S1590-8658(04)00095-7

[24] Tejero, F., Roschman-González, A., Perrone-Carmona, T.M. and Aso, P.M. (2008) Trypanosoma evansi: A Quantitative Approach to the Understanding of the Morphometry-Hematology Relationship throughout Experimental Murine Infections. The Journal of Protozoology Research, 18, 34-47.

[25] Perrone-Carmona, T.M., Garrizo, J., Roschman-González, A., Tejero, F., Escalante, A. and Aso, P.M. (2006) Susceptibility of Different Mouse Strains to Experimental Infection with a Venezuelan Isolate of Trypanosoma evansi. The Journal of Protozoology Research, 16, 1-8. 